\title{
Erratum: "Search for Gamma-Ray Emission from the Coma Cluster with Six Years of Fermi-LAT Data" (2016, ApJ, 819, 149)
}

M. Ackermann ${ }^{1}$, M. Ajello ${ }^{2}$, A. Albert ${ }^{3}$, W. B. Atwood ${ }^{4}$, L. Baldini ${ }^{3,5}$, J. Ballet ${ }^{6}$, G. Barbiellini ${ }^{7,8}$, D. Bastieri ${ }^{9,10}$, K. Bechtol ${ }^{11}$, R. Bellazzini ${ }^{12}$, E. Bissaldi ${ }^{13}$, R. D. Blandford ${ }^{3}$, E. D. Bloom ${ }^{3}$, R. Bonino ${ }^{14,15}$, E. Bottacini ${ }^{3}$, J. Bregeon ${ }^{16}$, P. Bruel ${ }^{17}$, R. Buehler ${ }^{1}$, G. A. Caliandro ${ }^{3,18}$, R. A. Cameron ${ }^{3}$, M. Caragiulo ${ }^{13,19}$, P. A. Caraveo ${ }^{20}$, J. M. Casandjian ${ }^{6}$, E. Cavazzuti $^{21}$, C. Cecchi $^{22,23}$, E. Charles ${ }^{3}$, A. Chekhtman ${ }^{24,58}$, G. Chiaro ${ }^{10}$, S. Ciprini ${ }^{21,22}$, J. Cohen-Tanugi ${ }^{16}$, J. Conrad ${ }^{25,26,55}$, S. Cutini ${ }^{21,22,27}$, F. D’Ammando ${ }^{28,29}$, A. de Angelis ${ }^{30}$, F. de Palma ${ }^{13,31}$, R. Desiante ${ }^{14,32}$, S. W. Digel ${ }^{3}$, L. Di Venere ${ }^{13,19}$, P. S. Drell ${ }^{3}$, C. Favuzzi $^{13,19}$, S. J. Fegan ${ }^{17}$, Y. Fukazawa ${ }^{33}$, S. Funk ${ }^{34}$, P. Fusco ${ }^{13,19}$, F. Gargano ${ }^{13}$, D. Gasparrini ${ }^{21,22}$, N. Giglietto ${ }^{13,19}$, F. Giordano ${ }^{13,19}$, M. Giroletti ${ }^{28}$, G. Godfrey ${ }^{3}$, D. Green ${ }^{35,36}$, I. A. Grenier ${ }^{6}$, S. Guiriec ${ }^{36,56}$, E. Hays ${ }^{36}$, J. W. Hewitt ${ }^{37}$, D. Horan ${ }^{17}$, G. Jóhannesson ${ }^{38}$, M. Kuss ${ }^{12}$, S. Larsson ${ }^{26,39}$, L. Latronico ${ }^{14}$, J. Li $^{40}$, L. Li $^{26,39}$, F. Longo ${ }^{7,8}$, F. Loparco ${ }^{13,19}$, M. N. Lovellette ${ }^{41}$, P. Lubrano ${ }^{22,23}$, G. M. Madejski ${ }^{3}$, S. Maldera ${ }^{14}$, A. Manfreda ${ }^{12}$, M. Mayer ${ }^{1}$, M. N. Mazziotta ${ }^{13}$, P. F. Michelson ${ }^{3}$, W. Mitthumsiri ${ }^{42}$, T. Mizuno ${ }^{43}$, M. E. Monzani ${ }^{3}$, A. Morselli ${ }^{44}$, I. V. Moskalenko ${ }^{3}$, S. Murgia ${ }^{45}$, E. Nuss ${ }^{16}$, T. Ohsugi ${ }^{43}$, M. Orienti $^{28}$, E. Orlando $^{3}$, J. F. Ormes ${ }^{46}$, D. Paneque ${ }^{3,47}$, M. Pesce-Rollins ${ }^{3,12}$, V. Petrosian ${ }^{3}$, F. Piron ${ }^{16}$, G. Pivato ${ }^{12}$, T. A. Porter ${ }^{3}$, S. Rainò ${ }^{13,19}$, R. Rando ${ }^{9,10}$, M. Razzano ${ }^{12,57}$, A. Reimer ${ }^{3,48}$, O. Reimer ${ }^{3,48}$, M. Sánchez-Conde ${ }^{25,26}$, C. Sgrò ${ }^{12}$, E. J. Siskind ${ }^{49}$, F. Spada ${ }^{12}$, G. Spandre ${ }^{12}$, P. Spinelli ${ }^{13,19}$, H. Tajima ${ }^{3,50}$, H. Takahashi ${ }^{33}$, J. B. Thayer ${ }^{3}$, L. Tibaldo ${ }^{51}$, D. F. Torres ${ }^{40,52}$, G. Tosti ${ }^{22,23}$, E. Troja ${ }^{35,36}$,

G. Vianello ${ }^{3}$, K. S. Wood ${ }^{41}$, S. Zimmer ${ }^{25,26}$ (i)

(The Fermi-LAT Collaboration), and

$$
\text { Y. Rephaeli }{ }^{53,54}
$$

${ }^{1}$ Deutsches Elektronen Synchrotron DESY, D-15738 Zeuthen, Germany

${ }^{2}$ Department of Physics and Astronomy, Clemson University, Kinard Lab of Physics, Clemson, SC 29634-0978, USA

${ }^{3}$ W. W. Hansen Experimental Physics Laboratory, Kavli Institute for Particle Astrophysics and Cosmology, Department of Physics and SLAC National Accelerator Laboratory, Stanford University, Stanford, CA 94305, USA

${ }^{4}$ Santa Cruz Institute for Particle Physics, Department of Physics and Department of Astronomy and Astrophysics, University of California at Santa Cruz, Santa Cruz, CA 95064, USA

${ }^{5}$ Università di Pisa and Istituto Nazionale di Fisica Nucleare, Sezione di Pisa I-56127 Pisa, Italy

${ }^{6}$ Laboratoire AIM, CEA-IRFU/CNRS/Université Paris Diderot, Service d'Astrophysique, CEA Saclay, F-91191 Gif sur Yvette, France

${ }^{7}$ Istituto Nazionale di Fisica Nucleare, Sezione di Trieste, I-34127 Trieste, Italy

${ }^{8}$ Dipartimento di Fisica, Università di Trieste, I-34127 Trieste, Italy

${ }^{9}$ Istituto Nazionale di Fisica Nucleare, Sezione di Padova, I-35131 Padova, Italy

${ }^{10}$ Dipartimento di Fisica e Astronomia "G. Galilei," Università di Padova, I-35131 Padova, Italy

${ }^{11}$ Dept. of Physics and Wisconsin IceCube Particle Astrophysics Center, University of Wisconsin, Madison, WI 53706, USA

${ }_{12}^{12}$ Istituto Nazionale di Fisica Nucleare, Sezione di Pisa, I-56127 Pisa, Italy

${ }^{13}$ Istituto Nazionale di Fisica Nucleare, Sezione di Bari, I-70126 Bari, Italy

${ }^{14}$ Istituto Nazionale di Fisica Nucleare, Sezione di Torino, I-10125 Torino, Italy

${ }^{15}$ Dipartimento di Fisica Generale "Amadeo Avogadro," Università degli Studi di Torino, I-10125 Torino, Italy

${ }^{16}$ Laboratoire Univers et Particules de Montpellier, Université Montpellier, CNRS/IN2P3, Montpellier, France

${ }^{17}$ Laboratoire Leprince-Ringuet, École polytechnique, CNRS/IN2P3, Palaiseau, France

${ }^{18}$ Consorzio Interuniversitario per la Fisica Spaziale (CIFS), I-10133 Torino, Italy

${ }^{19}$ Dipartimento di Fisica "M. Merlin" dell'Università e del Politecnico di Bari, I-70126 Bari, Italy

${ }^{20}$ INAF-Istituto di Astrofisica Spaziale e Fisica Cosmica, I-20133 Milano, Italy

${ }^{21}$ Agenzia Spaziale Italiana (ASI) Science Data Center, I-00133 Roma, Italy

22 Istituto Nazionale di Fisica Nucleare, Sezione di Perugia, I-06123 Perugia, Italy

${ }^{23}$ Dipartimento di Fisica, Università degli Studi di Perugia, I-06123 Perugia, Italy

${ }^{24}$ College of Science, George Mason University, Fairfax, VA 22030, USA

25 Department of Physics, Stockholm University, AlbaNova, SE-106 91 Stockholm, Sweden; conrad@ fysik.su.se, zimmer@fysik.su.se

${ }^{26}$ The Oskar Klein Centre for Cosmoparticle Physics, AlbaNova, SE-106 91 Stockholm, Sweden

${ }^{27}$ INAF Osservatorio Astronomico di Roma, I-00040 Monte Porzio Catone (Roma), Italy

${ }^{28}$ INAF Istituto di Radioastronomia, I-40129 Bologna, Italy

${ }^{29}$ Dipartimento di Astronomia, Università di Bologna, I-40127 Bologna, Italy

${ }^{30}$ Dipartimento di Fisica, Università di Udine and Istituto Nazionale di Fisica Nucleare, Sezione di Trieste, Gruppo Collegato di Udine, I-33100 Udine

${ }^{31}$ Università Telematica Pegaso, Piazza Trieste e Trento, 48, I-80132 Napoli, Italy

${ }^{32}$ Università di Udine, I-33100 Udine, Italy

${ }^{33}$ Department of Physical Sciences, Hiroshima University, Higashi-Hiroshima, Hiroshima 739-8526, Japan

${ }^{34}$ Erlangen Centre for Astroparticle Physics, D-91058 Erlangen, Germany

${ }^{35}$ Department of Physics and Department of Astronomy, University of Maryland, College Park, MD 20742, USA

${ }^{36}$ NASA Goddard Space Flight Center, Greenbelt, MD 20771, USA

${ }^{37}$ University of North Florida, Department of Physics, 1 UNF Drive, Jacksonville, FL 32224, USA

${ }^{38}$ Science Institute, University of Iceland, IS-107 Reykjavik, Iceland

${ }^{39}$ Department of Physics, KTH Royal Institute of Technology, AlbaNova, SE-106 91 Stockholm, Sweden

${ }^{40}$ Institute of Space Sciences (IEEC-CSIC), Campus UAB, E-08193 Barcelona, Spain

${ }^{41}$ Space Science Division, Naval Research Laboratory, Washington, DC 20375-5352, USA

${ }^{42}$ Department of Physics, Faculty of Science, Mahidol University, Bangkok 10400, Thailand

${ }^{43}$ Hiroshima Astrophysical Science Center, Hiroshima University, Higashi-Hiroshima, Hiroshima 739-8526, Japan

${ }^{44}$ Istituto Nazionale di Fisica Nucleare, Sezione di Roma "Tor Vergata," I-00133 Roma, Italy

${ }^{45}$ Center for Cosmology, Physics and Astronomy Department, University of California, Irvine, CA 92697-2575, USA

${ }^{46}$ Department of Physics and Astronomy, University of Denver, Denver, CO 80208, USA 


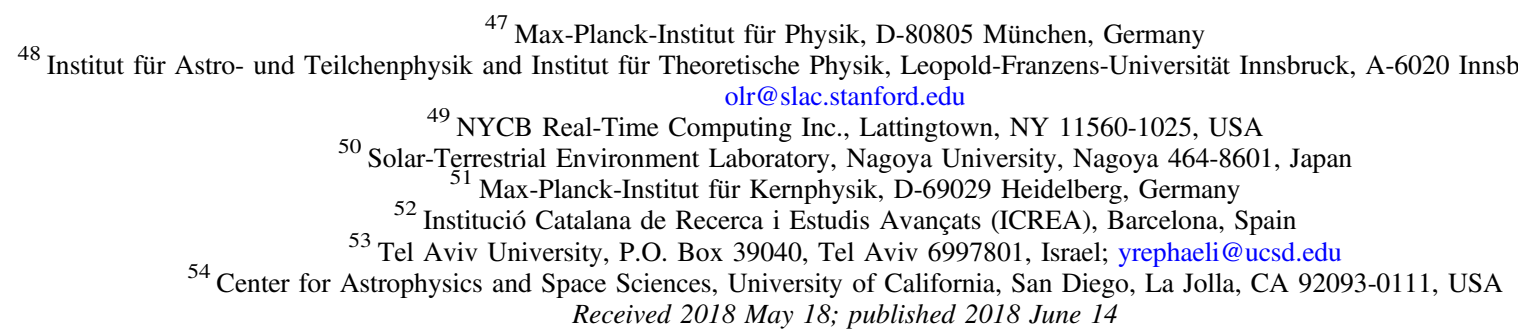

The naming of the sources in Tables 3 and 4 in the Appendix of the published paper did not follow IAU conventions; the corrected nomenclature will enter the NASA Extragalactic Database. Tables 3 and 4 should thus read as follows.

Table 3

Point Source Candidates in ROI

\begin{tabular}{lcccc}
\hline \hline Name & $\begin{array}{c}\text { R.A. } \\
\left({ }^{\circ}\right.\end{array}$ & $\begin{array}{c}\text { Decl. } \\
\left({ }^{\circ}\right.\end{array}$ & Model & Parameters \\
\hline xFGL J1241.6+3438 & 190.40 & 34.65 & PL & $\Gamma=1.88, E_{0}=3496 \mathrm{MeV}$ \\
xFGL J1250.9+3117 & 192.73 & 31.29 & LP & $\alpha=1.83, \beta=0.39, E_{b}=2407 \mathrm{MeV}$ \\
xFGL J1330.8+2933 & 202.70 & 29.55 & LP & $\alpha=2.57, \beta=0.41, E_{b}=1237 \mathrm{MeV}$ \\
xFGL J1245.8+2048 & 191.45 & 20.80 & PL & $\Gamma=3.45, E_{0}=1000 \mathrm{MeV}$ \\
\hline
\end{tabular}

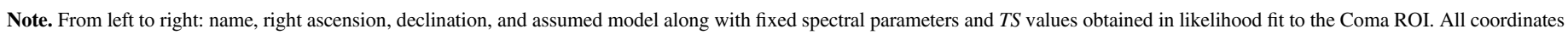
are given in $\mathrm{J} 2000$ epoch.

Table 4

Best-fit Parameters of the Background Model

\begin{tabular}{lc}
\hline \hline Source Name & $\begin{array}{c}\text { Normalization, } N_{0} \\
\left(\mathrm{~cm}^{-2} \mathrm{~s}^{-1} \mathrm{MeV}^{-1}\right)\end{array}$ \\
\hline 3FGL J1230.3+2519 & $(2.11 \pm 0.09) \times 10^{-12}$ \\
3FGL J1231.7+2847 & $(0.93 \pm 0.04) \times 10^{-12}$ \\
3FGL J1254.5+2210 & $(1.10 \pm 0.10) \times 10^{-13}$ \\
3FGL J1258.1+3233 & $(1.40 \pm 0.10) \times 10^{-12}$ \\
3FGL J1258.4+2123 & $(0.30 \pm 0.10) \times 10^{-12}$ \\
3FGL J1301.5+3333 & $(0.29 \pm 0.05) \times 10^{-12}$ \\
3FGL J1303.0+2435 & $(2.80 \pm 0.10) \times 10^{-12}$ \\
3FGL J1310.6+2446 & $(0.35 \pm 0.09) \times 10^{-13}$ \\
3FGL J1310.6+3222 & $(2.60 \pm 0.05) \times 10^{-11}$ \\
3FGL J1314.8+2349 & $(0.53 \pm 0.03) \times 10^{-12}$ \\
3FGL J1321.0+2215 & $(4.20 \pm 0.10) \times 10^{-12}$ \\
3FGL J1323.0+2942 & $(0.60 \pm 0.03) \times 10^{-12}$ \\
3FGL J1326.1+2931 & $(0.40 \pm 0.30) \times 10^{-14}$ \\
3FGL J1332.8+2723 & $(0.66 \pm 0.09) \times 10^{-12}$ \\
xFGL J1245.8+2048 & $(1.50 \pm 0.20) \times 10^{-13}$ \\
xFGL J1241.6+3438 & $(1.40 \pm 0.40) \times 10^{-14}$ \\
xFGL J1250.9+3117 & $(0.60 \pm 0.10) \times 10^{-13}$ \\
xFGL J1330.8+2933 & $(2.10 \pm 0.40) \times 10^{-13}$ \\
Extragalactic Diffuse $^{\mathrm{a}}$ & $(1.04 \pm 0.01)$ \\
Galactic Diffuse & $(1.04 \pm 0.02)$ \\
\hline
\end{tabular}

\section{Note.}

${ }^{\text {a }}$ The fitted value corresponds to the overall (unit-less) normalization of an all-sky template. The nominal value is 1.0.

\section{ORCID iDs}

\section{S. Zimmer 누 https://orcid.org/0000-0002-5735-0082}

\footnotetext{
${ }_{55}^{5}$ Wallenberg Academy Fellow.

56 NASA Postdoctoral Program Fellow.

57 Funded by contract FIRB-2012-RBFR12PM1F from the Italian Ministry of Education, University and Research (MIUR).

58 Resident at Naval Research Laboratory, Washington, DC 20375, USA.
} 